$\mathrm{H} 2 \mathrm{Av}$, the main marker of DNA breaks, on telomeres after HeT-A derepressing, suggesting that HeT-A overexpression could cause DNA breaks in telomeres. Moreover, the presence of DNA breaks in telomeres was accompanied by the appearance of R-loops, the DNA-RNA hybrid structures associated with DNA damage. Chromatin immunoprecipitation was done to prove the accumulation of R-loops in telomeres. The formation of R-loops is most likely caused by retention of HeT-A transcripts in chromatin. Thus, telomere-associated RNA is an essential factor of telomere stability during normal oogenesis and early development.

Program for Molecular and Cellular Biology of Presidium of Russian Academy of Sciences

doi: http://dx.doi.org/10.7124/bc.0009FD

\section{S-9. PEV-induced HP1a propagation does not correlate with the expression of the genes located near the eu- heterochromatin breakpoint}

\author{
A. A. Solodovnikov, S. A. Lavrov, \\ V. A. Gvozdev \\ Institute of molecular genetics RAS, 123182 \\ Kurchatov sq. 2, Moscow, Russia \\ Gorbins@gmail.com
}

Position effect variegation (PEV) is a disturbance of the expression of euchromatic genes transferred into the heterochromatin vicinity caused by the changes in its chromatin organization (heterochromatinization). Little is known about the molecular mechanisms of interactions between gene transcription machinery and the large-scale chromatin structures like heterochromatin, and the chromosomal rearrangement In(2)A4 provide a convenient model to study PEV. The aim of our work was to track the changes in chromatin organization of euchromatin in the vicinity of $\operatorname{In}(2) A 4$ new eu-heterochromatin borders and analyze the possible correlations between chromatin changes and the functional organization of the affected regions. Methods: We've performed analysis of genome-wide HP1a distribution in $\operatorname{In}(2) \mathrm{A} 4 /$ In(2)A4 homozygous flies and in the control wild type flies by ChIP-Seq with qPCR verification and bioinformatic analysis of the received data. Results: $\operatorname{In}(2) \mathrm{A} 4$ rearrangement is an inversion in the left arm of chromosome 2 with a breakpoint in the satellite block in the $2 \mathrm{~L}$ pericentromeric heterochromatin. This results in two new eu-heterochromatin boundaries - one near the main block of $2 \mathrm{~L}$ heterochromatin and another one near the separated small heterochromatin block. ChIP-Seq data on HP1a distribution shows an enrichment for HP1a in the euchromatin regions near the new eu-heterochromatin borders. HP1a spreads up to 200 $\mathrm{kb}$ from the main pericentromeric block and up to $50 \mathrm{~kb}$ from the small block. No apparent correlation between HP1a enrichment and genes expression levels (studied in [1]) or gene amenability to PEV were detected. The unusual enrichment in HP1a immediately near the small separated heterochromatin block was observed. Conclusions: In In(2)A4, HP1a propagates at a distance of up to $200 \mathrm{~kb}$ from the breakpoints and there is no apparent correlation between HP1a enrichment and expression levels of genes in the affected region as well as no correlation between HP1a binding and sensitivity of any particular gene to heterochroma- 
tin repression. It seems that HP1a propagation occurs independently of local chromatin organization defined by regulatory elements.

This work is supported by RFBR (grants 17-04-01984, 17-00-00282) and RSCF 19-74-20178

References 1. Abramov, Y.A., et al., The Differences Between Cis- and Trans-Gene Inactivation Caused by Heterochromatin in Drosophila. Genetics, 2016. 202(1): p. 93-106.

doi: http://dx.doi.org/10.7124/bc.0009FE

\section{S-10. DNA repair toolbox - cellular ability to repair multiple simultaneously induced single-strand DNA breaks}

\author{
Oskar Szelest, Damian Kurleto, Jurek \\ W. Dobrucki \\ Department of Cell Biophysics, Faculty of Bio- \\ chemistry, Biophysics and Biotechnology, Jagiello- \\ nian University, Krakow \\ szelest.oskar@gmail.com
}

Cellular capacity to repair DNA damage is limited, partly due to a fact that the number of copies of nuclear proteins available for a task of DNA repair is limited. Two key players involved in repair of SSBs, XRCC1 (X-ray Repair Cross Complementing Protein 1), which is involved in short patch and long path repair of singlestrand DNA breaks (SSBs), and PCNA (Proliferating Cell Nuclear Antigen), which is primarily involved in DNA replication, are recruited to the site of damage. Aims. This research is focused on understanding the process of saturation of a capacity to repair single-strand DNA breaks, by means of imaging recruitment of XRCC1 and PCNA to DNA lesions induced in non-replicating and replicating cells. The goal of this work was to assess cellular capacity to recruit detectable numbers of PCNA and XRCC1 molecules to several DNA lesions induced in close succession or separated by time interval, and quantitate a process of saturation of repair capacity as a function of the number of damage sites and the position of a cell in the cell cycle. Methods. Local DNA damage (SSBs) was induced by exposing a small region of the cell nucleus to a focused beam of laser light 1 . Live cells, expressing GFP-PCNA and RFP-XRCC1, or cells stained by immunofluorescence, were imaged using confocal fluorescence microscopy. Results. XRCC1 was recruited to the induced DNA lesions in all phases of the cell cycle, in contrast to PCNA which was not recruited in early S phase. Recruitment of PCNA was detected exclusively in middle and late S-phase. When DNA SSBs were induced at short time intervals (seconds) in several locations (from 1 to 30 ) in the cell nucleus, RFP-XRCC1 was recruited to only 5-6 of them. The recruitment of GFP-PCNA was limited to an even lower number of damage spots. When DNA lesions were induced in close succession, the amount of XRCC1 was the highest in the first spot, and lower in each subsequent location, suggesting that the cell activated recruitment very quickly, and was significantly exhausting the stock of the repair protein by recruiting it to each subsequent DNA lesion. Interestingly, a small amount of XRCC1 and PCNA which was not recruited to any damage site always remained in the cell nucleus outside of the damaged regions. Immunofluorescence studies confirmed the results obtained with live cells expressing fusion proteins. Conclusions. Cellular ability to repair single-strand DNA breaks that were generated 